\title{
Reflexion Sur Les Enjeux Epistemologique Et Methodologique De L'approche Anthropologique Sur La Maladie Chronique En Contexte Africain A Travers L'exemple Du VIH/Sida
}

\author{
Kouame Atta \\ Anthropologue biologiste, Enseignant-Chercheur, \\ Université Félix Houphouët Boigny de Cocody-Abidjan, Cote d'Ivoire
}

Doi: 10.19044/esj.2017.v13n29p344 URL:http://dx.doi.org/10.19044/esj.2017.v13n29p344

\begin{abstract}
Our practical experience of anthropology in the field of HIV/AIDS and many researchers' commitment in such scientific programs showed that social sciences' approach of chronic disease in African cultural context includes relevant epistemological and methodological issues. The purpose of this article is to give an overview of these issues while showing the contribution of the anthropological approach to the comprehension of the health phenomena related to the AIDS in the African environment. The approach to this was to review the literature on fieldwork already done on HIV / AIDS in the social sciences. The results of our analysis indicate that medical research on HIV / AIDS in African cultural context has mobilized social sciences mainly anthropology in its various specialties which alongside Biology, Epidemiology and Clinic have led to the development of an inclusive approach of the disease. This interactive approach between social sciences and medical sciences allowed fulfilling the limits of medicine's quantitative approaches and thus to grasp the AIDS pandemic in these epidemiological, behavioral and representational aspects.
\end{abstract}

Keywords: Anthropological approach, HIV/AIDS, epistemological and methodological issues, African context.

\section{Resume}

Notre pratique du terrain en anthropologie dans le champ du VIH/Sida et l'engagement de nombreux chercheurs dans de tels programmes scientifiques ont permis de comprendre que l'approche des sciences humaines sur la maladie chronique en contexte culturel africain comporte des enjeux épistémologique et méthodologique pertinents. L'objectif de cet article est de donner un aperçu de ces enjeux tout en montrant la contribution de 
l'approche anthropologique à la compréhension des phénomènes de santé liés au sida en milieu africain. La démarche pour y arriver, a consisté à examiner la littérature sur des recherches de terrain déjà effectuées sur le $\mathrm{VIH} /$ Sida dans le domaine des sciences humaines. Les résultats de notre analyse indiquent que la recherche médicale sur le VIH/Sida en milieu africain a mobilisé les sciences humaines plus principalement l'anthropologie dans ses différentes spécialités, qui, au côté de la biologie, de l'épidémiologie et de la clinique, ont donné lieu au développement d'une véritable approche globalisante de la maladie. Cette approche interactive entre sciences humaines et sciences médicales a permis de combler les limites des approches quantitatives de la médecine et de l'épidémiologie et ainsi saisir la pandémie du sida dans ces aspects épidémiologiques, comportementaux et représentationnels

Mots clés : Approche anthropologique, VIH/SIDA, Enjeux épistémologique et méthodologique, contexte africain.

\section{INTRODUCTION}

L'approche des Sciences humaines des problèmes de santé liés au $\mathrm{VIH} /$ sida en contexte africain comporte des enjeux épistémologique et méthodologique pertinents pour une analyse globalisante de cette pandémie. Le but de cet article est de donner un aperçu de ces enjeux à travers la contribution de l'anthropologie à la compréhension des phénomènes de santé liés au sida en milieu africain.

Les études sur le $\mathrm{VIH} /$ sida dans le monde, tout comme en Afrique, ont été, dès le départ, monopolisées par les sciences biologiques et médicales orientées vers l'examen et l'interprétation cliniques et numériques des phénomènes liés à l'infection (étude quantitative). Elles étaient, pour la plupart, des études étiologiques et épidémiologiques. L'une des raisons serait que l'engagement mondial en faveur de la lutte contre l'expansion de cette pandémie dans le monde et sur le continent africain nécessitait de telles études préalables. En effet, ces types d'études sont les seuls capables de produire des données en matière de prévalence, d'incidence, de prédiction, de causes ou d'effets et de généraliser d'un point de vue statistique les conclusions. Données statistiques qui sont nécessaires pour connaitre l'ampleur de l'épidémie et élaborer un protocole de sa prise en charge médicale. Ces programmes de recherches qui ont longtemps prévalu sont moins capables d'appréhender tous les contours d'une maladie comme le sida dont les comportements humains sont un facteur largement incriminé dans sa propagation. Les comportements qui sont un frein à la lutte contre le $\mathrm{VIH} / \mathrm{Sida}$ et favorables à sa propagation sont assez complexes surtout en Afrique pour être appréhendés par des études purement biomédicales. C'est à 
juste titre que les sciences biomédicales et les institutions internationales ont initié les enquêtes CACP (Connaissances, Attitudes, Croyances et Pratiques), RP (Relations entre Partenaires) en vue de combler l'analyse anthropologique de la prévention et de la prise en charge du sida en Afrique sans en assumer clairement et pleinement la perspective méthodologique (Vidal, 1995). Vidal fait remarquer que, «face à l'urgence [...] constituée par la progression du sida, la médecine recherche des analyses et des appréciations sur les comportements sexuels (contextes psychologiques, culturels ou économiques les favorisant) et les effets de l'épidémie sur les structures familiales (leurs capacités à prendre en charge la maladie), sans pour autant accepter les méthodes en mesure de produire ces données dans une perspective anthropologique impliquant une démarche qualitative, inscrite dans la durée et fondée sur l'observation et l'échange avec des interlocuteurs précis ». L'approche que préconise l'OMS initiateur de ces enquêtes, était plutôt quantitative répondant mieux aux finalités pratiques et épidémiologiques des résultats. Pour rattraper ce retard de l'analyse et de la perspective anthropologique de la prévention et de la prise en charge du sida, l'UNESCO et l'ONUSIDA ont initié en 1998 un projet conjoint intitulé l'approche culturelle de la prévention et du traitement du VIH/SIDA, dont le but est de mettre en relief la nature complexe de la maladie et la nécessité d'élaborer des stratégies multidimensionnelles de sa prise en charge. Cette approche représente une nouvelle contribution à la recherche de solutions au défi de la lutte contre la pandémie en milieu africain. Son atout méthodologique qui repose sur la démarche des sciences humaines consiste à adapter le contenu et le rythme de l'action aux mentalités, aux croyances, aux systèmes de valeurs et aux capacités de mobilisation des populations et à modifier en conséquence les stratégies et les politiques nationales et internationales de santé en matière du Sida (Unesco et Onusida, 2003). Cette initiative répond clairement aux principes et aux orientations de la Déclaration d'engagement sur le VIH/SIDA adoptée à l'issue de la session extraordinaire de l'Assemblée générale des Nations Unies (ONU) sur le VIH/SIDA (juin 2001), qui souligne « le rôle important que peuvent jouer la culture, la famille, les valeurs morales et la religion dans la prévention de l'épidémie et dans les activités de traitement, de soins et d'appui, compte tenu des particularités de chaque pays et de la nécessité de respecter tous les droits de l'homme et les libertés fondamentales » (paragraphe 20). Les maladies chroniques comme le sida comportent certes une dimension clinique, étiologique et épidémiologique qui relève des études médicales, mais comporte une autre dimension plus complexe (relative aux comportements à risque, à la représentation et au contexte socioculturel de la maladie, au vécu et aux implications socio-sanitaires de la maladie), qui est mieux accessibles à l'approche des sciences humaines (Casebeer et Verhoef, 
2000 ; Hudelson, 2004). En milieu culturel africain particulièrement, cette complexité est plus grande en raison des logiques explicatives de la maladie (Essi, 2002) et des représentations traditionnelles de la sexualité encore très marquées. Ces phénomènes complexes en milieu africain sont plus appréhendables par l'approche qualitative des sciences humaines (Borgès Da Silva, 2001), plus précisément l'Anthropologie. Casebeer et Verhoef (op cit.) font clairement ressortir la nécessité d'intégrer une approche des sciences humaines à l'étude des maladies chroniques. Pour eux, afin de comprendre et gérer ces maladies aux effets sans cesse plus débilitants, il faut regrouper les connaissances diverses accumulées au fil du temps pour améliorer qualitativement que quantitativement, le temps qui reste à vivre aux personnes atteintes. Seules les initiatives de recherches qui combinent des démarches et des compétences diverses parviennent à mieux faire comprendre comment gérer au mieux ces maladies. Comme le dit si bien Cyrulnik, "aucun problème humain n'a de réponse unique. Il faudra s'entraîner à donner la parole à diverses disciplines... » (2001).

Nos travaux de terrain depuis notre engagement dans le champ du $\mathrm{VIH} /$ sida résumés dans les paragraphes ci-après et de nombreuses publications sur le Sida en milieu africain de collègues des sciences humaines (Laurent Vidal, Alice Desclaux, Karine Delaunay, Fred Eboko, Jean Pierre Dozon, Blibolo Didier, Fatoumata Ouattara, Philippe Msellati, Olivier Nay, pour ne cité que ceux-là) éclairent la complexité des phénomènes comportementaux et socio-sanitaires liés à cette infection inaccessibles à l'approche numérique biomédicale. Tous ces éclairages appellent à une approche des sciences humaines, dans notre cas, de l'anthropologie sur le sida en contexte africain. Cette approche renvoie à l'examen et l'interprétation non numériques d'observations (non accessible à l'étude médicale), qui permet de découvrir les explications sous-jacentes des phénomènes complexes liés à l'infection et à sa prise en charge. Elle est nécessaire pour deux raisons essentielles intimement liées: la rigueur méthodologique dans l'appréhension et la rigueur épistémologique pour la compréhension des phénomènes complexes liés au sida en milieu africain. C'est à l'élucidation de ces deux principales raisons que nous nous attèlerons dans les lignes qui suivent à travers l'analyse des données produites dans nos différentes recherches et celles de collègues sur le $\mathrm{VIH} /$ sida en milieu africain.

\section{DEMARCHE METHODOLOGIQUE}

Ce texte est un article théorique qui rend compte des résultats d'études de terrain, en sciences humaines, réalisée sur le VIH en milieu africain. La démarche a consisté donc à examiner et à analyser sous un angle nouveau la littérature sur des recherches empiriques déjà effectuées sur le VIH/Sida dans 
le domaine des sciences humaines afin de mettre en évidence l'intérêt épistémologique et méthodologique de telles recherches. Notre analyse s'appuie essentiellement sur les résultats de nos travaux de recherche et sur ceux de collègues des sciences humaines portant sur le VIH/Sida en contexte africain. C'est une étude qualitative phénoménologique qui mobilise l'analyse de contenu et l'approche interprétative comme techniques d'analyse. Cette approche phénoménologique accorde de l'importance à l'essence ultime des choses, à l'intentionnalité et au sens que les acteurs sociaux donnent aux évènements qu'ils vivent (Husserl cité par N'Da, 2002 p 54). Il s'agit plus précisément de dépasser l'approche objectivante et d'accorder la primauté à la connaissance subjective de la souffrance liée à la maladie chronique et plus spécialement au VIH/Sida. Cette démarche nous a conduit à établir des catégories significatives donnant lieu à la structuration de notre discussion.

\section{ENJEUX EPISTEMOLOGIQUE ET METHODOLOGIQUE DES ETUDES ANTHROPOLOGIQUES SUR LE SIDA EN MILIEU AFRICAIN.}

Pour mieux analyser ces enjeux, il nous faut tout d'abord examiner de près la démarche des sciences médicales et ses limites afin de déduire les phénomènes complexes de la maladie qu'elle ne peut appréhender et qui relèvent du domaine des sciences humaines.

\section{Démarche des sciences médicales à l'étude du VIH/Sida et ses limites}

Dans l'étude des maladies en général et du VIH/Sida en particulier, les sciences médicales procèdent par une approche épidémiologique, clinique et numérique. Avec cette approche, nous constatons chaque jour que la maladie résulte d'un ensemble de causes objectives et qu'en recherchant ces causes, l'on peut agir et la guérir. Avec elle, nous voyons la maladie comme un fait objectif, c'est-à-dire ce qu'elle est d'un point de vue matériel et concret. Et pourtant ! Celui qui apprend à rencontrer le malade, dans notre cas le «malade » du VIH/Sida en milieu africain, constate bien vite que cette démarche des sciences médiales a des limites, qu'elle ne permet pas de comprendre l'ensemble des problèmes que la maladie, va poser au «malade». La mort, l'angoisse, les peurs, la souffrance, le manque et le désespoir liés à la maladie, son vécu, les relations du «malade » avec l'entourage, sont des problèmes humains qui ne peuvent pas se résoudre dans une simple connaissance objective de la maladie. La souffrance psychique qui accompagne la maladie chronique est un problème qui échappe aux études épidémiologiques et à leur approche.

Une autre dimension négligée par l'approche numérique des études sur le sida est la diversité culturelle des causes de la maladie en contexte 
africain. En effet, dans la conception stéréotypée des Africains, les causes et les origines de la maladie sont d'abord supranaturelles: une action maléfique d'un tiers, une transgression d'un interdit ou d'un totem, un nonrespect grave de la parole donnée. Le corps qui supporte les douleurs n'est donc qu'un médiateur physique (N'Guessan, 1978). Cette réalité de l'humain africain fait croire à une diversité des manifestations symptomatiques de la maladie chronique et voudrait que les recherches sur la maladie en contexte africain prennent cette dimension en compte pour pouvoir faire une lecture totale des signes observés dans la manifestation du comportement pathologique.

Les variables subjectives liées à ces phénomènes complexes sur le sida qui ne peuvent être appréhendées pertinemment par les sciences médicales situent tout l'intérêt de l'apport des sciences humaines et plus précisément de l'Anthropologie ...dont nos recherches et certaines de nos collègues chercheurs illustrent bien nombre des aspects.

\section{Nos productions en anthropologie dans le champ du VIH/sida.}

Depuis notre engagement dans le champ du VIH/Sida à l'occasion des études doctorales, nous avons enregistré une somme de recherches de terrain qui illustrent des enjeux épistémologique et méthodologique de l'approche anthropologique sur la maladie chronique en milieu culturel africain. Les recherches doctorales (Kouamé, 2009) se sont inscrites dans une approche bio-culturelle de l'étude des comportements suicidaires de personnes souffrant de maladies chroniques, précisément le VIH/Sida, et ont permis de répondre à deux préoccupations majeures jusque-là négligées par l'approche biomédicale. Elles contribuent à combler le vide documentaire relatif à l'étude des répercussions biopsychologiques liés au VIH/Sida et du risque suicidaire chez le malade africain; et interpelle sur la nécessité d'une prise en charge complète de la personne infectée en tenant compte, dans les itinéraires thérapeutiques, au-delà du suivi médical, l'accompagnement psychologique. Les résultats auxquels, elles ont aboutis indiquent la présence, de conduites suicidaires chez les personnes vivant avec le VIH/sida à Abidjan, allant des idées suicidaires aux tentatives de suicide. Ces conduites sont déterminés par des facteurs biopsychiques (anxiété, dépression) liés à l'infection et à l'état de santé des infectés et par des facteurs psychosociaux liés au vécu de la maladie et à la qualité des rapports sociaux avec l'entourage (stéréotypies, stigmatisation, ostracisme, rejet social). Il s'agit d'une nécessaire incursion dans le champ de la souffrance psychique liée au VIH/Sida moins accessible aux études épidémiologiques et cliniques.

Poursuivant dans ce même champ, deux études postdoctorales (Kouamé, 2010 ; Kouamé, 2012) mettent également en exergue l'intérêt de l'approche anthropologique sur la maladie chronique en contexte africain. 
La première porte sur le vécu de l'infection sous le regard de l'entourage en contexte social africain dont l'intitulé exact est «Vivre avec le VIH sous le regard des autres en milieu africain : l'expérience de séropositifs rencontrés à Abidjan ». Cette étude, exclusivement qualitative, est basée sur l'analyse de témoignages de séropositifs et sur une revue documentaire de la question. Elle fait apparaitre clairement que les rapports des infectés avec l'entourage familial et immédiat impactent et entravent considérablement le traitement et le suivi médical. Ils sont victimes de divers maux dont la stigmatisation, la mise à l'index, l'isolement social, l'auto-isolement, le deuil d'enfantement. Cette réalité du monde des séropositifs est un obstacle à l'observance thérapeutique et à une lutte efficace contre la pandémie en milieu africain.

La seconde est une étude d'analyse théorique, méthodologique et pratique et porte sur l' «Approche psycho-qualitative du VIH/sida en contexte africain : cas du vécu de l'infection chez des patients du Service des maladies infectieuses et tropicales (SMIT) d'Abidjan ». Elle est une contribution à l'approche qualitative de l'étude des phénomènes psychocomportementaux liés au VIH en contexte africain. Elle a procédé essentiellement par l'analyse de contenu de témoignages recueillis auprès des patients et a permis de relever deux défis majeurs liés au contexte africain dans l'évaluation du VIH/sida. Le premier d'ordre théorique et méthodologique se situe sur le plan de l'approche. En effet, le VIH/sida comprend une souffrance somatique qui relève des études biomédicales, étiologiques et épidémiologiques de type quantitatif et une souffrance morale, beaucoup plus complexe liée au vécu de la maladie et à ses représentations, relevant, elle, des recherches qualitatives. En Afrique, le vécu lié au VIH/sida présente une spécificité marquante en raison du pronostic vital de cette maladie, des problèmes thérapeutiques (précarité du système sanitaire, pénurie de médicaments) et de la forte pression sociale (représentations du VIH/sida, clichés négatifs, place de l'enfantement dans les cultures africaines). L'emploi de l'approche qualitative dans son évaluation en milieu africain est donc un meilleur moyen de faire reculer les limites des connaissances sur cette infection. Le second est d'ordre social. Il se situe sur le plan de la prise en charge des malades et se justifie par la double souffrance (physique et psychologique) liée à cette maladie. Compte tenu du vécu lié au VIH/sida, particulièrement marqué en milieu africain, une surveillance psychologique est à prescrire dans les itinéraires thérapeutiques actuels afin de réussir une prise en charge complète des personnes vivant avec l'infection dans ce milieu, et spécifiquement à Abidjan.

Au-delà de ces orientations épistémologiques et méthodologiques de nos recherches qui illustrent des enjeux de l'Anthropologie du sida en 
contexte africain, celles de collègues travaillant sur la pandémie en milieu africain sont d'autres exemples à citer.

\section{Publications d'autres chercheurs illustrant les enjeux de l'Anthropologie du sida en milieu africain}

Celles-ci sont assez nombreuses, cependant quelques unes retiennent notre attention à titre illustratif dans cette rubrique.

Dans un ouvrage sur l'Anthropologie du sida en Afrique publié en 1996, Vidal fait constater qu'il est nécessaire pour la recherche médicale sur le Sida en Afrique de réévaluer ses méthodes et ses concepts à la lumière des caractéristiques de l'épidémie. Au terme de quatre années passé auprès de malades fortement touchées par le sida à Abidjan, l'auteur se propose d'engager une réflexion sur la capacité de l'anthropologie à penser le malade dans ses relations sociales : qu'il s'agisse de réfléchir sur les rapports du patient au médecin, les représentations que le malade se fait de son affection, de l'intervention des médecins et de l'attitude de ses proches, ou d'analyser, les choix des praticiens en matière d'annonce de la séropositivité. Tout ceci traduit un dispositif de silence autour d'une maladie qui pose la question du sens de la contamination et du rapport à autrui. Dans cet ouvrage, il entreprend de dévoiler la richesse des réponses que chacun apporte à la maladie. Le lien qui unit ces silences à des systèmes de représentations dynamiques traduit un des défis majeurs de la vie du séropositif : maîtriser le silence afin d'accepter le sens de la maladie.

Dans un autre ouvrage sur les rapports des femmes au sida en Afrique publié en 2000, le même auteur (Vidal), commente la manière dont les deux premiers cas de sida recensés au Zaïre avant la médiatisation de la maladie ont été décrits dans les rapports médicaux, ainsi que les sous-entendus sociaux de ces rapports. Du suivi qu'il a fait de quatre femmes abidjanaises (l'une séropositive, l'autre séronégative et les deux autres ne connaissant pas leur statut sérologique) et à partir d'une approche historique et anthropologique il découpe en trois périodes les discours et les représentations des femmes ou sur les femmes en ce qui concerne l'infection par le VIH: avant l'arrivée de l'épidémie ; lors de la première décennie du sida; depuis 1990 où apparaissent des signes de changement. A la seconde période, c'est-à-dire la première décennie du sida, les femmes africaines étaient désignées comme responsables de la propagation du VIH. L'exercice auquel l'auteur se donne lui a permis de dégager, à partir de l'analyse des récits de femmes, mais aussi et surtout des récits de femmes qui ne sont pas nécessairement séropositives, enceintes ou prostituées, les logiques sociales et les représentations qui définissent les rapports sociaux qui liés les femmes en temps de sida en Afrique mais également ceux qui lient les femmes et les hommes. Où l'autre est indexé comme porteur et vecteur du virus. 
Laurent Vidal n'hésite pas dans l'une de ces publications parue en 2004, de mettre en relation, d'une part, les réponses à la maladie, les rapports au risque et à l'autre, qu'il soit proche, souffrant ou savant, et, d'autre part, l'attitude du chercheur et les arguments méthodologiques et éthiques de l'Anthropologie rendant possible ce regard.

Desclaux et Raynaut (1997) quant à eux, insistaient sur le dépistage au VIH en Afrique au sud du Sahara, région fortement touchée par le sida, qui est une nécessité de santé publique et du conseil qui doit accompagner le dépistage. Pour eux, cette pratique, nouvelle pour les professionnels de la santé, comprend une part d'information personnalisée et un dialogue qui évaluera la situation du consultant par rapport au VIH, lui permettant de faire face à l'annonce du statut sérologique du patient et l'aidera à trouver un soutien auprès des services sanitaires et sociaux.

Desclaux et Desgrées du Loû (2006) ont fait une publication inédite, sur les femmes africaines face à l'épidémie du sida, qui résumait que la féminisation du sida en Afrique se situe à différents niveaux : (i) elles sont les plus touchées du fait que la vulnérabilité et le risque à l'infection sont plus élevés chez elles par rapport aux hommes ; (ii) ce sont elles qui assument plus de responsabilité dans la prise en charges des malades du sida et des soins; (iii) elles sont mieux dépistées et mieux traitées que les hommes à cause du dépistage systématique lors des consultations prénatales. Rentrant plus en détail, ces auteurs évoquent la plus grande vulnérabilité biologique d'exposition des femmes et le fait qu'elles n'ont pas toujours la capacité de négociation qui leur permettrait d'insister sur l'utilisation de préservatifs ou sur la fidélité de leur partenaire. En Afrique, l'enfantement reste une priorité chez les femmes même quand elles sont infectées. Cela augmente le risque de donner naissance à un enfant lui aussi infecté, doublé de celui de laisser des enfants orphelins. Les femmes africaines sont considérées comme des «soignants naturels » à domicile. Ceci représente pour elles, une charge supplémentaire dans la famille. Les soins apportés par la femme à un membre de la famille représente aussi une charge psychologique, "d'autant que les modes de transmission du virus sont encore largement méconnus et sa contagiosité souvent surestimée. Pour éviter le rejet, une femme doit en permanence gérer le secret. Elle doit aussi faire face au risque de transmission dans la famille, sans compter qu'ellemême peut être infectée, tomber malade et mourir».

L'originalité de ces études, que ce soit les nôtres comme celles des collègues, est leur démarcation des études quantitatives de types biomédicales sur le VIH et la prise en compte des phénomènes psychologiques et socioculturelles liées à l'infection; phénomènes qui échappent à l'analyse épidémiologique et clinique de l'infection. Il apparaît clairement dans ces recherches qu'il est nécessaire d'aborder l'étude du VIH/Sida en milieu 
Africain sous un angle nouveau largement conditionné par la double souffrance qui l'accompagne et le contexte du milieu socio-culturel assez complexe des malades. Ceci nécessite un remaniement épistémologique et méthodologique des approches.

\section{L'approche anthropologique, un modèle d'approche à l'étude du VIH/sida en Afrique.}

Comme le souligne si bien Verhoef et Casebeer (1997) l'adhésion à des paradigmes différents et distincts peut amener les chercheurs de disciplines différentes à penser qu'il n'existe qu'une seule façon authentiquement «scientifique» de mener des recherches. Or celui qui observe de près la maladie chronique et ses manifestations se rend vite compte que sa nature même, suppose «l'emploi complémentaire de méthodes de recherche aussi bien qualitatives que quantitatives puisqu'il s'agit à la fois de quantifier l'efficacité des traitements et de qualifier l'expérience de la maladie au cours de son évolution» [Holman cité par Verhoef et Casebeer (2000)]. Les distinctions habituelles entre les approches quantitative et qualitative se situent à différents niveaux.

$\begin{array}{ll}\begin{array}{l}\text { Concepts associés à l'approche } \\ \text { quantitative }\end{array} & \begin{array}{l}\text { Concepts associés à l'approche } \\ \text { qualitative }\end{array} \\ \begin{array}{l}\text { Type de raisonnement } \\ \text { Déduction }\end{array} & \begin{array}{l}\text { Type de raisonnement } \\ \text { Induction } \\ \text { Objectivité }\end{array} \\ \text { Cause } & \text { Subjectivité } \\ \text { Type de question } & \text { Explication } \\ \text { Prédéterminée } & \text { Type de question } \\ \text { Axée sur les résultats } & \text { Ouverte } \\ \text { Type d'analyse } & \text { Axée sur le processus } \\ \text { Estimation numérique } & \text { Type d'analyse } \\ \text { Inférence statistique } & \text { Description narrative } \\ \end{array}$

Différences entre recherche quantitative et qualitative d'après Verhoef et Casebeer (2000).

L'Anthropologie tout comme les sciences sociales et humaines s'est engagé dans des programmes scientifiques pour comprendre la maladie, en étudier les représentations, saisir les logiques d'exposition au risque, rendre compte de l'expérience des malades, analyser les politiques de prévention et de traitement (Delfraissy et Fassin, 2010, Préface P IX). Le malade comme personne, sa culture, son vécu, ses ressentiments, son histoire de vie, ses rapports avec sa famille, ses représentations de la maladie, ses craintes, ses projets, ses relations avec l'entourage et avec les soins, sont autant de domaines qui intéressent donc l'anthropologie. Disons avec ces auteurs que pour les sciences sociales en général et l'Anthropologie en particulier, 
l'épidémie " a ouvert de nouvelles voies de recherche : sur le risque et le stigmate, sur les sexualités et les identités, sur la souffrance et la solidarité, sur les soins aux autres et le souci de soi, sur les politiques sanitaires et les mouvements sociaux, sur les recherches thérapeutiques et les essais cliniques, sur les droits des malades et la présence des proches ». Le sida a révélé plus qu'aucune autre circonstance la nécessité pour les spécialistes des sciences humaines de clarifier la nature et les conditions de leur implication dans les études de santé. Le sentiment de l'urgence, la pression des patients, la sollicitation des soignants, l'interpellation des biologistes et des épidémiologistes, tout concourt à les placer devant une sorte d'obligation d'engagement dont eux-mêmes ressentaient la nécessité (ibidem).

Pour ce faire ils mobilisent l'approche qualitative adaptée à l'étude et l'interprétation des variables subjectives liées la souffrance morale de la maladie chronique. Les méthodes qualitatives comme les observations, l'entrevue (les entretiens), les histoires de vie, les inventaires documentaires constituent pour l'essentiel l'approche de l'anthropologie. Dans ce cas, il ne s'agit pas spécifiquement de vérifier des hypothèses prédéfinies mais de décrire et d'apprécier des comportements humains (Pagezy et Sevin, 1992), culturels ou sociaux d'une façon fine et détaillée dans le but de situer leur diversité et leur variabilité. L'observation participante (Emerson, 1981) permet au fil de la vie quotidienne à la fois de mener une approche de l'intérieur et de l'extérieur, de corriger les discours obtenus au cours d'entretiens individuels ou collectifs. Comme le souligne A. Hubert (1990) cité par Pagezy et Sevin (1992), le temps et la maîtrise de la langue et de la culture sont les facteurs de réussite essentiels devant être associés aux méthodes qualitatives.

L'anthropologie procède donc par observation minutieuse des phénomènes à étudier et par induction. Elle exclut les déductions et les généralisations et considère que chaque phénomène culturel ou bio-culturel est spécifique en son genre. C'est la science de la différence. Elle considère que chaque fait, insignifiant soit-il peut avoir une influence sur la manifestation d'un phénomène social ou comportemental. Pour cela sa démarche est une démarche holistique prenant en compte tous les aspects du phénomène du plus signifiant au moins signifiant.

La démarche des sciences humaines et particulièrement de l'Anthropologie, nous permet de percevoir l'être humain (le malade donc) comme un ensemble plus ou moins intégré et cordonné de différents niveaux d'organisation vibratoire : le soma siège de nos activités métaboliques, la psyché siège de nos émotions, nos sentiments et ressentiments, nos croyances, nos représentations, nos valeurs... 


\section{CONCLUSION}

L'implication des sciences humaines et plus particulièrement de l'Anthropologie à l'étude des maladies chroniques se justifie doublement. La maladie chronique s'accompagne d'une souffrance physique ou somatique, mais également d'une souffrance psychique. Seule, l'approche biopsychosociale de l'Anthropologie, qui rationalise l'analyse conjuguée des facteurs biologiques, psychologiques et sociaux de la maladie, permet de déceler leurs manifestations et de les prendre en compte. De même les phénomènes complexes liés à la maladie de nature comportementale, sociosanitaire et culturelle ne sont accessibles qu'aux sciences humaines et sociales. De ce fait, celles qui s'intéressent au champ du VIH/Sida en Afrique devront relever deux défis majeurs: l'un théorique et épistémologique et l'autre méthodologique.

Au niveau épistémologique, elles devront tenir compte de la double souffrance qui accompagne cette infection et élaborer de nouveaux concepts appropriés différents de ceux utilisés dans le discours des sciences biomédicales.

Enfin au niveau de la démarche, elles devront introduire dans leur approche des dimensions telles que l'expérience subjective (nécessitant des outils qualitatifs) et la finalité des comportements (le sens que les acteurs donnent à leurs conduites). Dimensions qui échappent aux études biomédicales mais qui sont essentielles à une véritable compréhension du $\mathrm{VIH} / \mathrm{Sida}$ et des comportements des acteurs impliqués dans sa prise en charge en Afrique.

\section{References:}

1. Borgès Da Silva, Georges $2001 \mathrm{La}$ recherche qualitative : un autre principe d'action et de communication. Revue médicale de l'assurance maladie 32 (2) : 117-121.

2. Casebeer, Ann L. \& Verhoef, Marija J. 1997 Broadening horizons: integrating quantitative and qualitative research. Can J Infect Dis 8 (2):665-6.

3. Casebeer, Ann L. et Verhoef, Marija J 2000 L'utilisation combinée des méthodes de recherche qualitatives et quantitatives pour améliorer l'étude des maladies chroniques. In Maladies chroniques au canada, Volume 18 (3).

4. Cyrulnik, Boris 2001 Manifeste pour la résilience. ERES Spirale $18: 77-82$.

5. Desclaux, Alice et Raynaut, Claude. (dir.) 1997 Le dépistage du VIH et le conseil en Afrique au sud du Sahara, Paris, Karthala. Desclaux, Alice et Desgrées du Loû, Annabel (2006) «Les femmes africaines face à l'épidémie de sida », population et sociétés $n^{\circ} 428$. 
6. Emerson, Robert M. 1981 Observational field work. Annual Review of Sociology 7: 351-378.

7. Essi, Marie-José 2002 Sida et endogeinité : le sens du syndrome au sein d'un système local de santé. Les Cahiers de Mutations, 009, 9.

8. Fassin, Didier et Delfraissy, Fassin Didier 2010 Un engagement réfléchi In VIH/sida, se confronter aux terrains Expériences et postures de recherche. Autour de Fanny Chabrol et Gabriel Girard, dir. 185 p. IX-X. Paris : Collection sciences sociales et Sida.

9. Hudelson, Patricia. $2004 \mathrm{La}$ recherche qualitative en médecine de premier recours. Revue médicale suisse 503(24011). Repéré à http://revue.medhyg.ch/article.php3?sid=24011.

10. Kouamé, Atta. 2009 Conduites suicidaires chez les personnes vivant avec le VIH/Sida à Abidjan (Côte d'Ivoire) : Etude des facteurs de risque associés. Thèse Anthropobiologie. Universités de la Méditerranée (Aix-Marseille 2) et de Cocody (Abidjan), Marseille.

11. Kouamé, Atta 2010 Vivre avec le VIH sous le regard des autres en milieu africain : l'expérience de séropositifs rencontrés à Abidjan. Revue Africaine d'Anthropologie, Nyansa-Pô, $\mathrm{n}^{\circ}$ 10: 23- 40, Abidjan.

12. Kouamé, Atta. 2012 Approche psychoqualitative du VIH/sida en contexte africain : cas du vécu de l'infection chez des patients du Service des maladies infectieuses et tropicales (SMIT) d'Abidjan. Recherches Qualitatives 31(1) : 130-151. Québec.

13. N'Da, Paul 2002 Méthodologie de la recherche : de la problématique à la discussion des résultats, comment réaliser un mémoire, une thèse en Sciences sociales et en Education. Abidjan : EDUCI, 144 p.

14. N'Guessan, Koukou François, 1978 Pour une anthropologie médicale africaine. In Annales de l'Université d'Abidjan, Série F Tome VII ethnosociologie.

15. Pagezy, Hélène et Sevin, André. 1992 Approche méthodologique de l'anthropologie alimentaire. In Anthropologie de l'immigration. Autour de Gilles Boëtsch et Jean-Noël Ferrié, dir. Les Cahiers de l'Iremam 142, p p. 123-129. Publication sur OpenEdition Books : 10 janvier 2014

16. Unesco \& ONUSIDA. 2003 L'approche culturelle de la prévention et $\mathrm{du}$ traitement du VIH/sida. Projet de recherche Unesco/ONUSIDA, Manuels méthodologiques (série spéciale $n^{\circ} 4$ ), Paris : Unesco.

17. Vidal, Laurent 1995 L'anthropologie, la recherche et l'intervention sur le sida en Afrique. Enjeux méthodologiques d'une rencontre. Sciences sociales et santé 13, (2) : 5-27.

18. Vidal, Laurent 1996 Le silence et le sens : essai d'anthropologie du sida en Afrique, Paris : Anthropos, $228 \mathrm{p}$. 
19. Vidal, Laurent, 2000 Femmes en temps de sida. Expériences d'Afrique, Paris, PUF, 195 p.

20. Vidal, Laurent 2004 Ritualités, santé et sida en Afrique. Pour une anthropologie $d u$ singulier, IRD-Karthala, Coll. «Hommes et sociétés » $209 \mathrm{p}$. 